\title{
FREQUÊNCIA DE MARCADORES DE HEPATITE B EM GESTANTES DE PRIMEIRA CONSULTA EM CENTROS DE SAÚDE DE ÁREA METROPOLITANA, SÃO PAULO, BRAZIL.
}

\author{
Ester Cerqueira SABINO (1), Elvira Maria GUERRA (2), Isabel Takano OBA (1), Angela Maria Miranda SPINA (1) \& \\ Adejalde José VAZ (3)
}

\begin{abstract}
RESUMO
A hepatite $\mathrm{B}$ é doença de graves consequências quando adquirida no período neonatal. No entanto, a identificação das gestantes, com risco de transmitirem a infecção a seus filhos, pode prevenir essas consequências através da imunização, passiva e ativa, dos bebês logo após o nascimento. Foram estudadas 477 gestantes de primeira consulta atendidas no período de abril a outubro de 1988 nos oito Centros de Saúde da rede estadual que abrangem o subdistrito do Butantan, região oeste do município de São Paulo, considerada carente de infra-estrutura básica. As 477 amostras de soro foram ensaiadas quanto à presença do marcador anti-HBc total, que permite detectar os casos assintomáticos, crônicos e em fase de convalescença. As $44(9,2 \%)$ gestantes positivas para esse marcador foram ensaiadas para os marcadores anti-HBs e HbsAg. Dessas 44 amostras, $2(0,4 \%)$ foram positivas para o HBsAg e $37(7,7 \%)$ positivas para anti-HBs. Do universo de 477 gestantes, 47 apresentaram no inquérito a que foram submetidas respostas indicativas de fatores de risco para hepatite, mas apenas $8(17,0 \%)$ delas faziam parte do grupo de gestantes anti-HBc positivas, 2 delas $\mathrm{HBsAg}$ positivas.
\end{abstract}

UNITERMOS : Hepatite B; Gestantes; Anti-HBc; Epidemiologia.

\section{INTRODUÇÃO}

A hepatite $B$, causada pelo víns $B$ (HBV), vírus com DNA de dupla hélice, $42 \mathrm{~nm}$, da família Hepadnaviridae, constitue grave problema de Saúde Pública principalmente nas populações expostas a determinados fatores de risco.

O antígeno de superfície do vírus (HBsAg) é produzido em excesso e encontra-se circulante na forma de partículas de $22 \mathrm{~nm}$ esféricas ou tubulares, podendo ser encontrado no soro a partir de 10-14 dias após a exposição, e persistir por períodos variáveis.

O anticorpo anti-HBs passa a ser detectável após o controle da infecção e seu achado indica imunidade de longa duração.
Anticorpos anti-HBc (produzido contra antígeno do "core" ou $\mathrm{HBcAg}$ ) desenvolvem-se sempre e persistem por periodos muito longos.

Vários autores ${ }^{8,11,16}$ recomendam testes de triagem para anti-HBc com a finalidade de avaliar contato atual ou anterior com o vírus; sua fração IgM (anti-HBc IgM) é considerada um bom marcador da infecção recente ou aguda.

O terceiro antígeno, $\mathrm{HBeAg}$, é compreendido atualmente como um produto de clivagem enzimática do $\mathrm{HBcAg}$, sendo detectado em várias amostras de casos de infecção aguda ou crônica. Sua presença está relacionada com a replicação viral e alta infectividade, e o aparecimento de

(1) Instituto Adolfo Lutz - Laboratório de Hepatite - Divisão de Biologia Médica. Av. Dr. Arnaldo, 355, CEP 01246 São Paulo, SP, Brasil.

(2) Instituto Adolfo Lutz - Seção de Hematologia. Divisão de Patologia, São Paulo, SP, Brasil.

(3) Instituto Adolfo Lutz - Seção de Sorologia. Divisão de Biologia Médica, São Paulo, SP, Brasil. 
SABINO, E. C.; GUERRA, E. M.; OBA, I. T.; SPINA, A. M. M. \& VAZ, A. J. - Freqüência de marcadores de hepatite B em gestantes de primeira consulta em centros de saúde de área metropolitana, São Paulo, Brasil. Rev. Inst. Med. trop. S Paulo, 34 (6): 535-541, 1992.

anticorpos anti-HBe indica redução da replicação viral e da infectividade ${ }^{5,8,11}$.

A hepatite B é uma doença grave, e pelo fato de poder ser transmitida ao recém-nascido, adquire grande importância na Medicina perinatal. Acredita-se que $10 \%$ a $90 \%$ das mães positivas para antígeno HBs (HBsAg) transmitam a doença a seus recém nascidos ${ }^{1}$. Esta variação depende principalmente de presença do $\mathrm{HBeAg}^{1,3}$. Cerca de $90 \%$ das crianças infectadas no período neonatal irão evoluir como portadoras crônicas de $\mathrm{HBsAg}^{4}$, e, dessas, $25 \%$ irão apresentar complicaçð̃es graves como cirrose hepática e carcinoma hepatocelular na idade adulta ${ }^{6}$. Já as crianças de mães reagentes para $\mathrm{HBsAg}$ mas sem $\mathrm{HBeAg}$ têm um risco menor de adquirirem a infecção, embora casos de infecção aguda com hepatite fulminante fatal tenham sido relatados ${ }^{11,20}$.

A identificação de mulheres grávidas $\mathrm{HBsAg}$ positivas permite o tratamento dos recém-nascidos seja com o esquema de vacinação logo após o nascimento que previne a transmissão em 70 a $75 \%$ dos $\operatorname{casos}^{3,7}$, seja com o esquema de administrar soro imune anti-HBs (HBIG) e concomitante, ou posteriormente dentro de um mês, início do esquema de vacinação $0^{8}$. Tem sido recomendado que em 12 horas após o nascimento se administre via intramuscular $0,5 \mathrm{~mL}$ de $\mathrm{HBIG}$ e metade da dose usual da vacina. Este esquema tem uma eficiência de 85 a $95 \%$ em prevenir o desenvolvimento do estado de portador crônico ${ }^{2}$. A eficiência deste tratamento decresce com o passar do tempo, sendo mínima se a administração do soro imune for feita após 48 horas do nascimento 6 .

Recentemente os "Centers for Disease Control - CDC", têm recomendado, nos Estados Unidos, a triagem sorológica para deteç̧ão de $\mathrm{HBsAg} \mathrm{em}$ todas as mulheres grávidas com intuito de prevenir a transmissão vertical ${ }^{8,9}$.

LEE et al. ${ }^{13}$ detectaram HBsAg em $95 \%$ das amostras de secreção vaginal de mulheres infectadas e em apenas 35\% das amostras de líquido amniótico, corroborando com a hipótese de exposição mais comum no momento do parto ${ }^{8,13}$.

No Brasil, estudos de marcadores sorológicos de hepatite $B$ têm sido realizados principalmente em doadores de banco de sangue, na maioria do sexo masculino. Com exceção da região Norte onde a prevalência é alta, a frequência de positividade para o HBsAg tem variado entre 1 a $2 \%$, valores estes considerados baixos ou intermediários, segundo a Organização Mundial de Saúde .

A revisão bibliográfica quanto ao estudo de marcadores sorológicos em gestantes em nosso meio mostrou a escassez absoluta desse tipo de trabalho. SILVA et al., 1986, em Salvador, BA, estudaram 1382 soros pareados, das gestantes e do cordão umbilical dos respectivos recém-nascidos, quanto a presença de $\mathrm{HBsAg}$. A positividade entre as gestantes foi de $1,7 \%$ e a frequência de transmissão obșervada, de $27,3 \%{ }^{19}$.

Mudanças no comportamento sexual assim como o aumento do uso de drogas endovenosas podem estar alterando esta frequência em populações mais jovens.

A proposição deste trabalho é avaliar a frequência de marcadores sorológicos para hepatite B em uma população de gestantes de primeira consulta em Centros de Saúde da rede pública, no intuito de iniciar estudos visando avaliação da introdução deste exame na rotina do pré-natal, dada a gravidade da doença quando adquirida no período neo-natal.

\section{CASUÍSTICA E MÉTODOS}

\section{População estudada}

Foram estudadas 477 gestantes de primeira consulta, inscritas no Programa de Atendimento à Gestante de oito Centros de Saúde da Secretaria da Saúde do Estado de São Paulo, no subdistrito de Paz do Butantã, município de São Paulo, no período compreendido entre abril a outubro de 1988.

O subdistrito de Paz do Butantã está situado na zona oeste do município de São Paulo, onde se encontra grande número de favelas, contando com infraestrutura básica deficiente ${ }^{18}$.

Todas as gestantes que procuraram o serviço pela primeira vez, naquela gestação, nos Centros de Saúde: Escola Butantã, II Butantã, III São Luiz, II Rio Pequeno, II Caxingui, II Vila Borges, III Monte Kemel e II Real Parque, no periodo de abril a outubro de 1988, foram agendadas para a coleta de material, bem como para aplicação de inquérito ${ }^{12}$.

Foram colhidos $5 \mathrm{ml}$ de sangue venoso de 
SABINO, E. C.; GUERRA, E. M.; OBA, I. T.; SPINA, A. M. M. \& VAZ, A. J. - Freqüência de marcadores de hepatite B em gestantes de primeira consulta em centros de saúde de área metropolitana, São Paulo, Brasil. Rev. Inst. Med. trop. S Paulo, 34 (6): 535-541, 1992.

cada gestante, atendida pela primeira vez naquela gravidez, através do Sistema Vacutainer MR, em tubos siliconizados estéreis.

Na ocasião da coleta do sangue, foi aplicado um questionário a cada gestante com as seguintes informaçð̄es : idade, grupo étnico, ocupação, Estado de origem, idade gestacional, história de transfusão sanguínea, renda familiar e número de pessoas constituintes da família.

Na variável renda, foi utilizado o salário mínimo per capita (SMPC), que foi obtido através de duas divisões. Primeiro, foi dividida a renda familiar pelo piso nacional de salários do mês de aplicação do questionário. A seguir, a renda familiar em salários mínimos foi dividida pelo número de pessoas que compunham a família da gestante, inclusive as crianças, obtendo-se assim o SMPC (salários mínimos per capita). O valor do piso nacional de salário foi dividido pelo valor oficial do dólar americano no dia 30 de cada mês, obtendo-se assim, o piso nacional de salário em dólar.

\section{Critérios adotados}

Foram investigados os seguintes fatores de risco para hepatite: grupo étnico, história de transfusão, sorologia positiva para sífilis (FTA-abs), estado de origem e ocupação da gestante.

\section{Preparação dos soros}

Após duas horas a $25^{\circ} \mathrm{C}$, as amostras foram centrifugadas a $2.000 \mathrm{rpm}$ por 15 minutos. O soro obtido foi dividido em alíquotas de $0,5 \mathrm{ml}$, mantidas a $-20^{\circ} \mathrm{C}$ até a realização das provas sorológicas, que ocorreu no período de novembro de 1990 a janeiro de 1991. O aspecto do soro foi observado quanto à presença de lipemia e ictericia.

\section{Testes sorológicos}

Foram utilizados testes sorológicos com reagentes imunobiológicos comercialmente disponiveis no mercado.

Hepatite B - todas as amostras foram submetidas ao teste imunoenzimático para pesquisa de anticorpos anti-HBc Total (CORZYME ${ }^{\mathrm{M}}$, Abbott Lab, USA). As amostras positivas foram submetidas aos testes imunoenzimáticos para detecção de HBsAg e anti-HBs (Abbott Lab, USA).
Sífilis - para todos os soros foram realizados os testes VDRL (CELM, BR.) e FTA-abs (IMUNO-SERUM, BR.) ${ }^{22}$.

\section{Análise dos dados}

As frequências de positividade foram obtidas pelo programa "DBASE III PLUS" para microcomputador IBM PC compatível e testadas quanto às diferenças entre os grupos de risco e sem risco, utilizando-se o teste do Qui-quadrado. O nível de significância aceito foi igual ou menor que 0,05 $(p \leq 0,05)$.

\section{RESULTADOS}

A idade média das gestantes foi de 24,5 anos, com idade mínima de 14 e máxima de 46 anos. Das 477 gestantes, $229(48,0 \%)$ estavam no primeiro trimestre gestacional, $200(41,9 \%)$ no segundo e $48(10,1 \%)$ no terceiro.

Quanto ao grupo étnico, 252 (52,8\%) eram caucasóides, $224(47,0 \%)$ eram negróides e uma gestante $(0,2 \%)$ era mongolóide.

Em relação ao Estado de origem, $157(33,0 \%)$ eram nascidas no Estado de São Paulo, 59 (12,4\%) procedentes de outros Estados da região sudeste, $215(45,1 \%)$ de Estados do nordeste, $40(8,4 \%)$ de Estados do sul, $1(0,2 \%)$ da região norte, $4(0,8 \%)$ do centro-oeste e $1(0,2 \%)$ proveniente do Chile.

Nas famílias das gestantes foram consideradas as pessoas que efetivamente viviam no mesmo lar independente do parentesco. Em média essas familias tinham 4 pessoas. $O$ número mínimo foi de um, o número máximo foi de 15 pessoas na família, e $24(5 \%)$ gestantes não tinham família.

A renda média, calculada em SMPC, foi de 0,97 SMPC; 7 (1,5\%) não declararam renda, para $306(64,2 \%)$ a renda foi inferior a um SMPC; 149 $(31,2 \%)$ tinham de 1,00 a 2,99 SMPC e somente $15(3,1 \%)$ declararam renda de 3 ou mais SMPC. Em média, um piso nacional de salário no período de abril a outubro de '1988, correspondia a US\$ 52,3 , tendo variado de US\$ 50,1 a US\$ 53,5.

Em nenhuma das 477 amostras de soro foram observadas icterícia e/ou lipemia.

Das 477 gestantes, $44(9,22 \%)$ foram positivas 
SABINO, E. C.; GUERRA, E. M.; OBA, I. T.; SPINA, A. M. M. \& VAZ, A. J. - Freqüência de marcadores de hepatite B em gestantes de primeira consulta em centros de saúde de área metropolitana, São Paulo, Brasil. Rev. Inst. Med. trop. S Paulo, 34 (6): 535-541, 1992.

para anti-HBc, e destas, $2(0,42 \%)$ positivas para HBsAg, $37(7,75 \%)$ positivas para anti-HBs e 5 $(1,05 \%)$ apresentavam apenas o anti-HBc como marcador (Tabela 1).

Considerando os fatores de risco investigados, das 477 gestantes, $24(5,0 \%)$ apresentaram reação positiva para sífilis (FTA-abs), $1(0,2 \%)$ era mongolóide (origem oriental), $21(4,4 \%)$ apresentaram história de transfusão, $1(0,2 \%)$ era procedente da regiåo norte e $1(0,2 \%)$ gestante trabalhava na área de saúde (atendente de enfermagem).

Das gestantes com anti-HBc positivo ( $\mathrm{n}=44)$, a maioria, ( 36 ou $81,8 \%$ ), não apresentou qualquer fator de risco dentre os estudados. As outras 8 $(17,0 \%)$ apresentavam fatores de risco, sendo 3 com sorologia positiva para sífilis, $3 \mathrm{com}$ história de transfusão, 1 era procedente da região norte e 1 trabalhava na área da saúde. As 2 gestantes $\mathrm{HBsAg}$ positivas estavam neste grupo.

O grupo de gestantes com fator de risco $(n=47)$ apresentou maior número de gestantes anti$\mathrm{HBc}$ positivas $(17,0 \%)$ do que o grupo onde fatores de risco eram ausentes $(8,4 \%)$, sendo essa diferença estatísticamente significativa $(0,02<\mathrm{p}<0,05)$.

\section{DISCUSSĀO}

A infecção pelo HBV no curso da gravidez pode ter graves consequências para o neonato, mas podem ser previstas e evitadas.

MUSHAHWAR et al. ${ }^{16}$, em 1981, estudaram os marcadores sorológicos analisando amostras seriadas de 149 pacientes com hepatite $B$, cobrindo os períodos de incubação, sintomático, convalescença, recuperação e desenvolvimento da forma crônica. Os autores observaram que 79,0\% dos casos seguem o curso de um episódio agudo com total recuperação e $21,0 \%$ tomam-se portadores persistentes do $\mathrm{HBsAg}$, com evidência de cronicidade.

Pela observação dos resultados sorológicos os autores concluem que há distintos padrões de marcadores e resposta imune. De modo geral, a detecção de HBsAg e HBeAg e ausência de seus anticorpos indicam, respectivamente, infecção aguda recente e alta infectividade; o desaparecimento do $\mathrm{HBeAg}$ e a deteç̧ão concomitante de anticorpos anti-HBc e anti-HBe indicam fase de soroconversão do sistema e, na fase crônica podem, no entanto, persistir os antígenos circulantes .

A manutenção do HBsAg com o aparecimento do anti-HBc pode ocorrer em assintomáticos crônicos e durante infecções agudas. No último caso, coincide com o início da resoluçãa da doença ${ }^{16}$.

A detecção do marcador anti-HBc e ausência de HBsAg e seu anticorpo, pode ocorrer na convalescença, ou representar infeç̧ão passada. Os portadores crônicos, neste caso, teriam níveis de HBsAg e/ou HBeAg em níveis não detectáveis pelos testes em uso ${ }^{16}$.

Na fase de total recuperação com o estabelecimento de imunidade persistente, observam-se apenas os anticorpos, desaparecendo posteriormente, $o$ anti-HBe e o anti-HBc-IgM nesta ordem. Desse modo os pacientes considerados totalmente recuperados apresentam apenas anticorpos, frequentemente anti-HBs e anti-HBc. Os imunizados ativamente, apresentam apenas o anti-HBs, visto que foram imunizados apenas com as proteínas representativas do HBsAg.

Por fim MUSHAHWAR et al ${ }^{16}$ indicam a necessidade de se ampliar o número de testes quando se investiga a hepatite $B$ para que se possa definir - padrão sorológico dos marcadores e correlacioná-lo com a fase da doença.

\section{Tabela 1}

Distribuição das 477 gestantes segundo os resultados obtidos no teste sorológico para hepatite (anti $\mathrm{HBc}$ ) segundo as gestantes com fator de risco e sem fator de risco para hepatite.

\begin{tabular}{llll}
\hline & \multicolumn{2}{c}{ anti- HBc } & \\
\cline { 2 - 4 } Gestantes & $\begin{array}{c}\text { positivo } \\
\mathrm{n}(\%)\end{array}$ & $\begin{array}{c}\text { negativo } \\
\mathrm{n}(\%)\end{array}$ & $\begin{array}{c}\text { Total de gestantes } \\
\mathrm{n}(\%)\end{array}$ \\
\hline $\begin{array}{l}\text { COM fator } \\
\text { de risco }\end{array}$ & $8(17,0)$ & $39(83,0)$ & $47(100,0)$ \\
$\begin{array}{l}\text { SEM fator } \\
\text { de risco }\end{array}$ & $36(8,4)$ & $394(91,4)$ & $430(100,0)$ \\
\hline TOTAL & $44(9,2)$ & $433(90,8)$ & $477(100,0)$ \\
\hline
\end{tabular}

Análise do Qui-quadrado significativo.

Qui-quadrado calculado $=3,87^{*}$

Qui-quadrado crítico $=3,84$ 
SABINO, E. C.; GUERRA, E. M.; OBA, I. T.; SPINA, A. M. M. \& VAZ, A. J. - Freqüência de marcadores de hepatite B em gestantes de primeira consulta em centros de saúde de área metropolitana, São Paulo, Brasil. Rev. Inst. Med. trop. S Paulo, 34 (6): 535-541, 1992.

No presente trabalho foi utilizado o marcador anti-HBc total na triagem sorológica, o que permitiu detectar com segurança os casos assintomáticos, crônicos e em fase de convalescença. Não houve no grupo nenhuma amostra sérica ictérica. A frequência de contato prévio com o HBV foi de $9,2 \%$ (44/477), sendo que apenas 2 gestantes $(0,4 \%)$ mostraram evidência de infecção atual (positividade para $\mathrm{HBsAg}$ ).

Este último resultado é inferior ao observado por SILVA et al..$^{19}$, em 1986, que estudaram 1382 gestantes em Salvador, BA, e encontraram 23 $(1,7 \%)$ delas positivas para o marcador HBsAg.

Dentre as 42 amostras HBsAg negativas $88,1 \%$ (37/42) apresentaram positividade para o marcador anti-HBs. Já SILVA et al. ${ }^{19}$, em 1986, encontraram $34,6 \%$ de positividade para o antiHBs em 211 amostras HBsAg negativas. Além dos estudos serem realizados em populaçð̃es distintas, os resultados sugerem que análises dos marcadores sorológicos da hepatite $\mathrm{B}$ só podem ser comparáveis quando se define um perfil sorológico a partir de testes para os diversos marcadores, confirmando a opinião de MUSHAHWAR et al. ${ }^{16}, 1981$.

Na transmissão da hepatite B são reconhecidos como fatores de risco: origem oriental, história de hepatite, história de transfusão sanguinea, uso de drogas injetáveis, história de outras doenças sexualmente transmissíveis, constantes hemodiálises e atividade profissional na área da Saúde ${ }^{4,6,79,14,21}$.

No entanto, em cerca de $50 \%$ dos casos de portadores de $\mathrm{HBsAg}$ não se identifica nenhum desses fatores de risco ${ }^{21}$.

Neste trabalho em $81,8 \%$ das 44 gestantes com o marcador anti-HBc não foi possível detectar quaisquer dos fatores de risco estudados.

Nossos dados mostram uma frequência de HBsAg de $0,4 \%$ e de anti $\mathrm{HBc}$ de $9,2 \%$. Estes dados são semelhantes aos encontrados em Bancos de Sangue da cidade de São Paulo ${ }^{15}$. O Banco de Sangue da Santa Casa de Misericórdia de São Paulo encontrou em 11500 amostras, no ano de 1990, $1,6 \%$ de positividade para $\mathrm{HBsAg}$ e $9,4 \%$ de positividade para anti-HBc (comunicaçáo pessoal de CHIATTONE, C.S. - Banco de Sangue da Santa Casa de São Paulo ).
Entre as 477 gestantes aqui estudadas 47 apresentaram pelo menos um fator de risco associado para hepatite B. Apesar do número ser pequeno, os dois casos HBsAg positivos estavam neste grupo e seriam detectados em uma triagem onde apenas mulheres de risco fossem selecionadas, no entanto, das 47 gestantes com fatores de risco para hepatite apenas $8(17,0 \%)$ apresentaram positividade para anti-HBc.

No grupo de gestantes onde não foi observado nenhum fator de risco $(n=430)$, foram encontrados $8,4 \%$ de gestantes anti- $\mathrm{HBc}$ positivas, percentagem significativamente menor do que no grupo com fatores de risco $(0,02<p<0,05)$, resultados concordantes com a literatura9, ${ }^{914}$.

Nos Estados Unidos a frequência de HBsAg é semelhante a nossa, variando de cerca de $0,66 \%$ a $0,88 \%$. Inicialmente a triagem sorológica de gestantes para Hepatite $B$ naquele país estava restrita as mulheres com algum fator de risco ${ }^{6,7}$.

Recentemente SUMMERS et al. ${ }^{21}$ mostraram que $50 \%$ das gestantes HBsAg positivas não seriam detectadas utilizando o critério de prévia seleção das mulheres com história de fatores de risco.

No último boletim sobre contrôle das hepatites virais os "Centers for Disease Control (CDC)", em 1990 , têm recomendado a triagem universal das gestantes para hepatite $\mathrm{B}^{8}$.

SUMMERS et al ${ }^{21}$ estudaran as 15.399 gestantes atendidas no "Charity Hospital of Louisiana" em New Orleans de novembro de 1983 a outubro de 1985, e empregando o radioimunoensaio para detecção de HBsAg, encontraram 136 $(0,88 \%)$ pacientes com HBsAg detectável. Destas, em 108 foi investigada a presença de fatores de risco, encontrados em apenas $54(50,0 \%)$ delas, predominando a origem oriental em 39 e a história de hepatite em 8 delas.

O custo/benefício da triagem universal das gestantes foi calculado por SUMMERS et al. ${ }^{21} \mathrm{e}$ para a população estudada o valor foi semelhante ao da triagem universal de Bancos de Sangue.

CHRISTIAN \& DUFF', em 1989, estudaram prospectivamente 1520 gestantes atendidas no Madigan Army Medical Center no período de 1 ano (julho de 1987 a agosto de 1988), e encontra- 
SABINO, E. C.; GUERRA, E. M.; OBA, I. T.; SPINA, A. M. M. \& VAZ, A. J. - Freqüência de marcadores de hepatite B em gestantes de primeira consulta em centros de saúde de área metropolitana, São Paulo, Brasil. Rev. Inst. Med. trop. S Paulo, 34 (6): 535-541, 1992.

ram $10(0,66 \%)$ gestantes positivas para $\mathrm{HBsAg}$, todas de origem asiática $(n=186)$ e ao contrário de outros autores $^{8,21}$ sugerem a triagem dos pacientes por fatores de risco, o que reduziria em $70 \%$ o custo da triagem sorológica.

Na cidade de São Paulo, MINGIONE \& VIEIRA ${ }^{15}$, em 1991, estudando 3.777 doadores com idades entre 18 e 65 anos, no período de 01/ $10 / 90$ a 28/02/91, encontraram $241(6,3 \%)$ doadores positivos para anti-HBc, e $31(0,8 \%) \mathrm{HBsAg}$ positivos, sugerindo o marcador anti-HBc como o mais seguro na triagem de doadores de sangue.

MARTELLI et al. ${ }^{14}$ (1991) estudaram a soroprevalência de fatores de risco para as doenças infecciosas triadas em bancos de sangue de Goiania/GO, de outubro de 1988 a fevereiro de 1989. Dos 1033 doadores estudados, $132(12,8 \%)$ foram considerados já infectados pelo $\mathrm{HBV}, 20$ $(1,9 \%)$ com HBsAg positivo e $112(10,9 \%)$ com anti HBs. A história de hepatite ou icterícia indicou ser fator de risco de elevado valor preditivo.

O próposito do presente estudo foi apenas de iniciar uma discussão acerca da necessidade de se introduzir na triagem sorológica das gestantes atendidas na rede pública do Estado de São Paulo, testes que detectam marcadores da hepatite B.

Devido a limitaç̃es do método de coleta dos dados aqui apresentados, não podemos considerar o presente trabalho como um levantamento epidemiológico. No entanto, os resultados indicam a necessidade de continuidade neste tipo de estudo, inclusive pela escassez em nosso meio, particularmente em São Paulo, de publicaçð̄es semelhantes utilizando população de gestantes.

\section{SUMMARY}

Frequency of hepatitis B serological markers in first consultation pregnant women at health centers in a metropolitan area, $S$. Paulo city, Brazil.

Hepatitis B is a severe disease when acquired during the neonatal period. The identification of the infected pregnant women allows prevention of newborn infection by active and passive immunization soon after birth. We studied pregnant women in their first visit to eight different primary medical centers in Butantan, a subdistrict of S. Paulo city. 477 samples were tested for anti-HBc. From 44 (9.2\%) anti-HBc positive samples, $2(0.4 \%)$ were $\mathrm{HBsAg}$ positive and $37(7.7 \%)$ were anti-HBs positive. A risk factor for hepatitis B could only be detected in 8 (18.9\%) of the 44 anti- $\mathrm{HBc}$ positive samples.

\section{REFERÊNCIAS BIBLIOGRÁFICAS}

1. BEASLEY, R.P.; TREPO,C.; STEVENS, C.E. \& SZMUNESS, W.- The antigen and vertical transmission of hepatitis B surface antigen. Amer. J. Epidem.,105:94$98,1977$.

2. BEASLEY, R.P.; HWANG L.Y. \& LEE,G.G. - Prevention of perinatally transmitted hepatitis $B$ virus infection with hepatitis $B$ immune globulin and hepatitis $B$ vaccine. Lancet, 2: 1099-1102,1983.

3. BEASLEY, R.P.; HWANG, L.Y.; STEVENS, C.E.; LIN, C.C.; HSIEH, F.J.; WANG, K.Y.; SUN, T.S. \& SZMUNESS, W. - Efficacy of hepatitis B immune globulin for prevention of perinatal transmission of hepatitis B virus carrier state. Final report of a randomized doubleblind, placebo controlled trial. Hepatology, 3: 135-141, 1983.

4. BEASLEY, R.P.\& HWANG,L.Y. - Epidemiology of hepatocellular carcinoma. In : VYAS, G.N.; DIENTAG, I.L.\& HOOFNAGLE, J.H. Viral hepatitis and liver disease. New York, Grune \& Stratton, 1984. p.209-224.

5. CARRILHO, F.J. \& SILVA, L.C. - Epidemiologia. In: SILVA, LC. ed. Hepatites agudas e crónicas, São Paulo, Sarvier, 1986. p. 47-69.

6. CENTERS FOR DISEASE CONTROL - Recommendations for protection against viral hepatitis. J. Amer. med. Ass.,254:197-217, 1985.

7. CENTERS FOR DISEASE CONIROL - Prevention of perinatal transmission of hepatitis B virus. Prenatal screening of all pregnant women for hepatitis B surface antigen. MMWR,37: 341-346, 1988.

8. CENTERS FOR DISEASE CONTROL - Protection against viral hepatitis. Recommendations of the immunization practices advisory Committee (ACIP). MMWR, 39(S-2): 1-25, 1990.

9. CHRISTIAN, S.S. \& DUFF, P.- Is universal screnning for hepatitis $B$ infection warranted in all prenatal population? Obstet. and Gynec,74: 259-261, 1989.

10. DELAPLANE, D.; YOGEV, R.; CRUSSI, F. \& SCHULMAN, S.T.- Fatal hepatitis B in early infancy : the importance of identifying $\mathrm{HBsAg}$-positive pregnant women and providing immunoprophylaxis to their newboms. Pediatrics, 72: 176-180, 1983.

11. EDWARDS, M.S. - Hepatitis B serology - help in interpretation. Pediat. Clin. N. Amer.,35: 503-513, 1988.

12. GUERRA, E.M. - Prevalência de anemia em gestantes de primeira consulta em Centros de Saúde do Estado no subdistrito do Butantan, município de São Paulo. São 
SABINO, E. C.; GUERRA, E. M.; OBA, I. T.; SPINA, A. M. M. \& VAZ, A. J. - Freqüência de marcadores de hepatite B em gestantes de primeira consulta em centros de saúde de área metropolitana, São Paulo, Brasil. Rev. Inst. Med. trop. S Paulo, 34 (6): 535-541, 1992.

Paulo, 1989. (Dissertação de Mestrado - Faculdade de Ciências Farmacêuticas da USP).

13. LEE, A.K.Y; IP, H.M.H. \& WONG, V.C.W. - Mechanisms of matemal-fetal transmission of hepatitis $B$ virus. J. infect. Dis., 138: 668, 1978.

14. MARTELLI, C.M.T.; ANDRADE, A.L. S.S.; CARDOSO, D.D.P.; SILVA, S.A. \& ZICKER, F.Consideraçôes metodológicas na interpretação do rastreamento sorológico da hepatite $\mathrm{B}$ em doadores de sangue. Rev. Saúde pábl. (S. Paulo), 25: 11-16, 1991.

15. MINGIONE, C.J.G. \& VIEIRA, A. - Correlação entre marcadores de hepatite (HBsAg, anti-HBc e ALT) em doadores de sangue do Hospital A.C. Camargo. LAESHAES (S.Paulo), 72: 34-36, 1991.

16. MUSHAHWAR, I.K.; DIENSTAG, J.L.; POLESKY, H.F.; McGRATH, L.C.; DECKER, R.H. \& OVERBY, L.R. - Interpretation of various serological profiles of hepatitis B virus infection. Amer. J.clin. Path,76: 773777,1981 .

17. NISHIOKA, K. - Predominant mode of transmission of hepatitis $B$ virus : perinatal transmission in Asia. In: VYAS, G.N.; DIENSTAG, J.L. \& HOOFNAGLE, S.H., eds. Viral hepatitis and liver diseases. New York, Grune \& Stratton, 1984. p. 423-432.
18. SÃo PAULO, Secretaria da Saúde. Grupo de trabalho. Implantação de ERSA 2; relatório. São Paulo, 1986. (Mimeografado).

19. SIlVA, L; MOTTA, E.; NANO, M.J. \& LYRA, L.G.Transmissão vertical do AgHBs e passiva de anti-HBs em Salvador- Bahia. In: JORNADA LATINOAMERICANA DE HEPATOLOGIA, $9^{\circ}$, São Paulo, 1986. Anais. p.121.

20. SINATRA, F.R.; SHAH, P.; WEISSMAN, J.Y.; THOMAS, D.W.; MERRITT, R.J. \& TONG, M.J. - Perinatal transmitted acute icteric hepatitis $B$ in infants born to hepatitis B surface antigen-positive and anti-hepatitis Bepositive carrier mothers. Pediatrics, 70: 557-559, 1982.

21. SUMMERS, P.R.; BISWAS, M.K.; PASTOREK II, J.G.; PERNOLL, M.L.; SMITH, L.G. \& BEAN, B.E.- The pregnant hepatitis B carrier : evidence favoring comprehensive antepartum screening. Obstet. and Gynec.,69: $701-704,1987$.

22. VAZ, AJ.; GUERRA, E.M.; FERRATTO, L.C.C.; TOLEDO, L.A.S. de \& NETO, R.S.A. - Sorologia positiva para sífilis, toxoplasmose e doença de Chagas em gestantes de primeira consulta em Centros de Saúde de área metropolitana, Brasil. Rev. Saúde públ.(S. Paulo), 24: 373-379, 1990.

Recebido para publicaçāo em 27/12/1991 Aceito para publicação em 15/10/1992 